\title{
Introduction
}

\section{Advances in rehabilitation for functional neurological disorder}

\author{
Victor W. Mark \\ University of Alabama at Birmingham Birmingham, 619 19th Street South, SRC 190, Birmingham 35249, AL, \\ USA. Tel: +1 205934 3499; Fax: +1 205975 4524. E-mail: vwmark@uabmc.edu
}

Functional neurological disorder (FND) has mystified clinicians since antiquity, owing to its intermittent disturbances within the (usually) alert, attentive patient, as well as its lack of defining biomarkers following routine instrumented assessments (structural brain MRI in the individual patient at clinical magnetic field strengths, electroencephalography). Consequently, the etiology for FND (if there is only one) has not been conclusive, in contrast for many other common neurological disorders that are commonly associated with objective biomarkers. For example, focal structural lesions evident on the individual patient's brain or spinal structural MRI at conventional magnetic field strengths are often associated with characteristic, relatively stable neurological deficits; epileptic seizures coincide with characteristic and distinctive episodic waveforms on electroencephalography.

Adding to the mystique of FND is its common (though not invariant) association with mood disorders. The aggravation of FND following the patient's attention to the symptoms, and amelioration with distraction, have commonly suggested to clinicians that FND manifests some sort of a psychiatric disorder, and thus not deserving serious management in the fields of neurology and rehabilitation. Moreover, the presentations of FND can be convincingly simulated by healthy individuals (e.g., hemiparesis, tremor). As a result, this observation often impels clinicians to conclude that such patients are malingering for secondary gain. The resolution of FND, at least temporarily, with psychotherapy further suggests to many clinicians that FND is not actually a neurological disorder.

The net result is that persons with FND commonly feel marginalized by conventional health care practitioners and shunned by society. Often the patients report that they have been dismissively told, "It's all in your head," which shames the patients when it is implied to them that their problems could dissipate with simple persuasion.

However, it would be hard to name another illness that has been more under- or mis-treated. At present the majority of clinicians who may initially evaluate persons with FND are not trained for its management. One should, however, recognize that many observations of FND suggest that it is truly a neurological disorder: (1) Persons with FND are desperate for treatment. Individuals who are truly malingering would not likely go through the arduous process of serial evaluations and attempts at therapy, bouncing from practitioner to practitioner, as occurs with persons with FND. (2) A few reports have indicated acute severe neurological deficits in domesticated animals, following physical or emotional trauma, that completely reversed through behavioral intervention. This argues against a process of psychiatric "conversion" of symptoms that originate from early life trauma and subsequent suppression of unconscious experiences. Moreover, some of the leading characteristics of FND, particularly episodic immobility or non-combative hyperkinetic movements, occur commonly as anti-predator behaviors in terrestrial vertebrates or avians, either to simulate death 
(to deter would-be predators that typically avoid feasting on dead potential prey) or mimic being lame (to distract predators from the nest). These are instinctive, non-learned behaviors that have survival value, a consequence of natural selection. This raises the possibility that similar involuntary phenomena can occur in some humans as well, following acute emotional or physical trauma. (3) A few studies have shown that either FND patients or neurologically healthy individuals, when asked to simulate specific neurological deficits, have altered cerebral cortical physiological activation patterns that are distinct from the patterns in FND patients when they are not prompted to produce their neurological symptoms. (4) Numerous structural brain MRI scan studies likewise have demonstrated significantly abnormal structures (either regional expansion or atrophy) among persons who are diagnosed with FND. These anomalies are not evident in the individual but emerge from statistical quantitative averaging of structural brain MRI signals at the group level.

It is necessary to demystify FND. The disorder deserves firmly to be placed in the panoply of neurological disorders. The six papers in this issue respond to the need to further the understanding of FND and methods for their management, and thus help to make FND knowable.

The first two papers critically review the definition and diagnosis of FND. Kirkwood and Mark look comprehensively at the inclusion criteria of studies over the past 20 years on the objective biomarkers of FND, and find that there is no gold standard. Instead, there is about an equal application of three different diagnostic methods: either those that were developed for assessing "functional" dystonia; the criteria provided in the Diagnostic and Statistical Manual of "Mental" Disorders (DSM) editions; or individual clinician-determined diagnoses (including from non-specialists) that did not follow specific guidelines. Kirkwood and Mark conclude that the lack of standardized diagnostic guidelines for FND limits characterizing biomarker signatures of FND and methods for treatment. Consequently, it is necessary to standardize the diagnostic criteria for FND before moving on to characterize its biomarkers and test its rehabilitation in clinical trials. Mark in turn comprehensively reviews the clinical characteristics of "legacy" neurological disorders (i.e., those not considered to be FND) and finds considerable overlap with the features of patients who are considered to have FND. Mark finds that the distinction between legacy disorders vs. FND are so unclear that the need to diagnose a specific form of illness as "FND" cannot be supported. In response, Mark introduces the alternate diagnostic term "attentionally-modifiable disorder." He recommends that clinicians to be alert for symptoms that can be modified by the patient's self-attention, regardless of the disease's characteristics, and when finding such, to refer the patients for behavioral therapy. The term "attentionally-modifiable disorder" is recommended to co-exist with biomarker-based diagnostic terms (e.g., stroke, brain tumor), and the biomarker aspects of such disease should be managed according to disease-specific practice standards.

The next two papers present case reports to showcase the efficacious rehabilitation methods for motor FND. These case reports are remarkable for being among the few that are accompanied by video demonstrations before and after straightforward rehabilitation. Geary presents three women with functional gait disorders who had fluctuating lower extremity control during examination. The treatments were efficacious through procedures to distract the patients from the motor deficit, encouragement, and graduating activities from simple to more complex in response to clinical improvement at each stage. The outcomes were highly beneficial. Hebb et al in turn present a complementary single case of functional gait disorder to focus on music therapy to guide functional recovery. Again, the outcome was positive. The reports together demonstrate the importance of empathetic respect for the patients' complaints, application of commonplace therapy techniques, and gradual advances of training activities from simple to more complex. These reports thus suggest that conventional rehabilitation therapy techniques and minimizing attention to deficits can be applied successfully to patients with functional motor disorder in general, and that these approaches deserve to be disseminated widely to other rehabilitation programs that have not attempted to treat FND.

The final two papers, both by Polich et al, review the feasibility and success of motor FND rehabilitation programs in the American healthcare system. The first describes the construction of the FND inpatient rehabilitation program at Spaulding Rehabilitation Hospital in Boston, USA, by rehabilitation specialists with expertise for caring for this disorder. The program was designed for a two-week stay involving multidisciplinary team care. Program development included staff education to lead to a coherent treatment approach. This report thus provides a blueprint by which other rehabilitation 
programs can prepare similar healthcare referral and management pathways. The other paper compares the experiences from three different motor FND rehabilitation programs in the United States. This illustrates the distinguishing features at several possible levels of healthcare complexity in response to the severity of the disease, ranging from outpatient physical therapy alone for milder symptoms to involving more disciplines and increased frequency of treatments, and finally to inpatient therapy for the most refractive or complex presentations. The emerging area of telehealth care for FND is also addressed, which is of increasing concern given that many patients have limited access to such care due to their being remote from specialized centers, and the need to adjust to the recurring waves of viral pandemics. Barriers toward establishing FND care pathways are reviewed, to allow developing FND care programs to apply the lessons that are described in this report.

The breadth of these reports reflects a broad array of concerns and experiences for the emerging field of medicine for a common but as yet incompletely treated neurological disorder. The authors hope that this thematic issue of NeuroRehabilitation will spur professional advance toward investigation, discussion, and management for this disorder, while enabling rehabilitation practitioners to gain familiarity, competence, and confidence with care for FND. 